\title{
CHARACTERIZATION OF THE EFFICIENCY OF THE FEATURES AGGREGATE IN FUZZY PATTERN RECOGNITION TASK
}

\author{
R. Grekov and A. Borisov \\ Specialized Institute of Intelligent Computer Technologies \\ Technical University of Riga \\ I, Kalkyu Street, Riga LV - 1658, Latvia \\ Phone: +3717320019 \\ Fax: +3717820094 \\ E-mail:aborisov@egle.cs.rtu.lv
}

\section{INTRODUCTION}

Let a set of objects exist each of which is described by $N$ features $X_{1}, \ldots, X_{N}$, where each feature $X_{i}$ is a real number. So each object is set by $N$-dimensional vector $\left(\mathrm{X}_{1}, \ldots, \mathrm{X}_{\mathrm{N}}\right)$ and represents a point in the space of object descriptions, $R^{N}$.

There are also set objects for which degrees of membership in either class are unknown. A decision rule should be determined that could enable estimation of the membership of either object with unknown degrees of membership in the given classes (Ozols and Borisov, 1996). To determine the decision rule, such features should be found which give a possibility to distinguish objects belonging to different classes, i.e. features that are specific for each class. That is why a subtask of estimation of the efficiency of features should be solved. A function $\delta$ should be determined which could enable estimation of the efficiency of both separate features and of features groups.

Thus, the task is reduced to the determination of a number of features from set $N$ that will best describe groups of objects and will enable possibly correct recognition of the object's membership in a class. 


\section{CHARACTERIZATION OF THIE EFFICIENCY OF A SINGLE FEATURE IN COMBINATION WYTH TWO OTHER FEATURES}

Let there exist a space of features $\mathrm{X} \times \mathrm{Y} \times \mathrm{Z} \times \mathrm{K}$. The efficiency of the aggregate of either of two features could be estimated through the composition of binary relations. For example, by taking compositions

\section{$X Z \circ Z Y$ and XZ०ZK}

it is possible to ascertain the influence of features $Y$ and $K$ in combinatioin with feature $Z$. In this case definitions that follow are valid: - negative synergy: importance of feature $Z$ in combination with feature $Y$ is not greater than that of feature $Z$ in combination with feature $K$. In other words, replacement of feature $K$ with feature $Y$, in combination with feature $Z$, will not improve recognition quality;

- positive synergy: importance of feature $Z$ in combination with feature $Y$ is greater than that of feature $Z$ in combination with feature $K$. In other words, the quality of recognition could be improved by substituting feature $Y$ for feature $K$, in combination with feature $Z$;

- independence: the middle case when there is no difference either feature $K$ or feature $Y$ is used. Recognition quality does not change.

\section{CHARACTERIZATION OF THE EFFICIENCY OF A PAIR OF FEATURES IN COMBINATION WITH TWO OTHER FEATURES}

Assume that a space of features $\mathrm{X} \times \mathrm{Y} \times \mathrm{Z} \times \mathrm{K}$ exists. By taking compositions of binary relations it is possible to characterize the efficiency of the aggregate of any features. For example, compositions of features

\section{$X Z \circ Z Y$ and $X Z \circ Z K$}

will enable us to ascertain the influence of the aggregate of features $Z$ and $K$ on the quality of recognition. Here, three definitions are valid:

- negative synergy: importance of a pair of features $Z$ and $K$, if they are taken in combination, is not greater than the sum of their individual importances. In other words, we will not be able to improve recognition quality by combining features;

- positive synergy: importance of a pair of features $Z$ and $K$, if they are taken in combination, is greater than the sum of their individual importances. In other words, the quality of recognition could be improved by combining features $Z$ and $K$; 
- independence: the middle case when each feature contributes to the quality of recognition.

\section{INTEGRAL ESTIMATION OF THE EFFICIENCY OF A FEATURE}

Assume, the efficiency of feature $Z$ should be estimated in three-dimensional space $X Y Z$. Let there exist shadows $S_{X Z}^{A}, S_{Z Y}^{B}, S_{X Z}^{B}$, $S{ }_{Z Y}^{A}$. Composition $X Z \circ Z Y$ is formed:

$$
\begin{aligned}
& \text { (1) } S_{X Z}^{A} \circ S_{Z Y}^{B} \text { and } \\
& \text { (2) } S_{X Z}^{B} \circ S_{Z Y}^{A} \text {. }
\end{aligned}
$$

As a result, we come to two reduction factors: $\delta_{X z}^{A}, \delta_{z Y}^{A}$ in the first case and $\delta_{X Z}^{B}, \delta_{Z Y}^{A}$ in the second one. Quantity $\delta_{X Z}^{A}$ characterizes the reduction over axis $Z$ with respect to class $A$ for composition (1), whereas $\delta_{z Y}^{B}$ characterizes reduction over axis $Z$ with respect to class $B$ for composition (1). Quantity $\delta_{x z}^{B}$ characterizes the reduction over axis $Z$ with respect to class $\mathrm{B}$ for composition (2) but $\delta_{Z Y}^{A}$ characterizes reduction over axis $Z$ with respect to class $A$ for composition (2). By taking a sum of those quantities over the classes $\left(\delta_{X Z}^{A}+\delta_{Z Y}^{A} ; \delta_{Z Y}^{B}+\delta_{X Z}^{B}\right)$ and taking then the difference of those sums, it is possible to obtain the importance of feature $Z$ as well as to ascertain which class is represented by feature $Z$ :

$\left(\delta_{x z}^{A}+\delta_{z Y}^{A}\right)-\left(\delta_{z Y}^{B}+\delta_{X Z}^{B}\right)$ : if $\left(\delta_{x z}^{A}+\delta_{z Y}^{A}\right)>\left(\delta_{z Y}^{B}+\delta_{x z}^{B}\right)$, feature $Z$ is the feature of class $A$;

$\left(\delta_{z Y}^{B}+\delta_{X Z}^{B}\right)+\left(\delta_{x Z}^{A}+\delta_{z Y}^{A}\right)$ : if $\left(\delta_{X Z}^{A}+\delta_{z Y}^{A}\right)<\left(\delta_{z Y}^{B}+\delta_{X Z}^{B}\right)$, feature $Z$ is the feature of class $\mathrm{B}$.

\section{REFERENCES}

Ozols Y. and Borisov A. A Comparative Analysis of the Features in the Fuzzy Pattern Classification. Proc. Fourth European Congress on Intelligent Techniques and Soft Computing, EUFIT' 96, Aachen, Germany, September 2-5, 1996, 1690-1694. 